\title{
Nonlinear Stability and Instability of Transonic Flows Through a Nozzle
}

T.-P. Liu

Department of Mathematics, University of Maryland, College Park, Maryland 20742, USA

\begin{abstract}
We study transonic flows along a nozzle based on a one-dimensional model. It is shown that flows along the expanding portion of the nozzle are stable. On the other hand, flows with standing shock waves along a contracting duct are dynamically unstable. This was conjectured by the author based on the study of noninteracting wave patterns. The author had shown earlier that supersonic and subsonic flows along a duct with various cross sections are stable. Basic to our analysis are estimates showing that shock waves tend to decelerate along an expanding duct and accelerate along a contracting duct.
\end{abstract}

\section{Introduction}

It is well known that gas flows are often highly unstable. This is clear from experimental studies and causes great difficulties in numerical calculations. However, the emergence of shock waves in the flow as a consequence of the nonlinearity of the gas dynamics equations makes it even more difficult for analytical studies. The present paper is a step in understanding such phenomena. We show that for a 1-dimensional model flows along an expanding duct are always asymptotically stable, while flows with a standing shock wave along a contracting duct are dynamically unstable. Since the initial value problem for the gas dynamics equations is expected to be well-posed, by instability we mean that the asymptotic state of a flow does not depend smoothly on the initial state.

The equations which model gas flow in a variable area duct are of the form [1]:

$$
\begin{gathered}
\frac{\partial \varrho}{\partial t}+\frac{\partial(\varrho u)}{\partial x}=-\frac{a^{\prime}(x)}{a(x)} \varrho u, \\
\frac{\partial(\varrho u)}{\partial t}+\frac{\partial\left(\varrho u^{2}+p\right)}{\partial x}=-\frac{a^{\prime}(x)}{a(x)} \varrho u^{2}, \\
\frac{\partial(\varrho E)}{\partial t}+\frac{\partial(\varrho E u+p u)}{\partial x}=-\frac{a^{\prime}(x)}{a(x)}(\varrho E u+p u),
\end{gathered}
$$

* $\quad$ Sponsored by the United States Army under Contract No. DAAG29-80-C-0041. This material is based upon work supported by the National Science Foundation under Grant No. MCS 7802202 and by the Sloan Foundation 
where $a(x)$ is the cross section of the duct, $\varrho, u, p$, and $E$ are, respectively, the density, velocity, pressure and the total energy of the gas, and $x \in R, t>0$. For a uniform duct, $a^{\prime}(x)=0$, the equations become

$$
\begin{gathered}
\frac{\partial \varrho}{\partial t}+\frac{\partial(\varrho u)}{\partial x}=0 \\
\frac{\partial(\varrho u)}{\partial t}+\frac{\partial\left(\varrho u^{2}+p\right)}{\partial x}=0 \\
\frac{\partial(\varrho E)}{\partial t}+\frac{\partial(\varrho E u+p u)}{\partial x}=0 .
\end{gathered}
$$

In this case it was shown by Liu [6] that the asymptotic state of a solution of the initial value problem consists of the elementary waves determined by the values of the initial data at $x= \pm \infty$. In particular, the flows are always asymptotically stable. The reason for such a strong stability phenomenon is that in spite of very complicated nonlinear wave interactions, waves also tend to combine, cancel and decouple as a result of the dependence of the characteristic speeds on the state and the hyperbolicity of the system. Consequently, a general solution tends to a wave pattern which is noninteracting. Such a wave pattern consists of shock waves, rarefaction waves and a traveling wave, and can be found by solving the Riemann problem which is an initial value problem with the initial data containing only two constant states:

$$
\begin{aligned}
\omega(x, 0) & =\left\{\begin{array}{lll}
\omega_{\ell} & \text { for } & x<0, \\
\omega_{r} & \text { for } & x>0,
\end{array}\right. \\
\omega & \equiv(\varrho, \varrho u, \varrho E),
\end{aligned}
$$

where $\omega_{\ell}$ and $\omega_{r}$ are constant states.

For a variable cross section duct, it has been shown by Liu [8] that supersonic and subsonic flows are also asymptotically stable provided that the duct becomes uniform as $x \rightarrow \pm \infty$. In this case, the asymptotic state consists of the aforementioned elementary waves and a standing wave which is a solution of

$$
\begin{aligned}
(\varrho u)_{x} & =-\frac{a^{\prime}(x)}{a(x)} \varrho u, \\
\left(\varrho u^{2}+p\right)_{x} & =-\frac{a^{\prime}(x)}{a(x)} \varrho u^{2}, \\
(\varrho E u+p u)_{x} & =-\frac{a^{\prime}(x)}{a(x)}(\varrho E u+p u) .
\end{aligned}
$$

When the flow is not transonic, shock waves and rarefaction waves travel with nonzero speed and eventually decouple from the standing wave. For transonic flows, these two kinds of nonlinear modes intertwine and more interesting nonlinear wave phenomena occur. Based on the above studies, one expects that a general solution also tends to an asymptotic state which does not create any nonlinear interaction. Such noninteracting wave patterns were studied in Liu [9]. It was found that for an expanding duct a noninteracting wave pattern is uniquely 
determined by its value at $x= \pm \infty$ and thus one should expect the general solution to be asymptotically stable. On the other hand, along a contracting duct there may exist three noninteracting wave patterns with the same given end states at $x= \pm \infty$. One wave pattern is smoothly deformed to a supersonic flow and the other to a subsonic flow. Thus these two should be stable by [8]. The third one, which contains a standing shock wave, is therefore expected to be unstable by the criterion of the exchange of stability. It is the purpose of this present paper to verify the above conjecture on the stability of wave patterns in the dynamical sense. In particular, we show that when a standing shock wave in a contracting duct is perturbed, it accelerates and the solution eventually tends to a stable wave pattern with no standing shock wave which differs greatly from the original unperturbed state. On the other hand, when a standing shock wave in an expanding duct is perturbed, it decelerates and tends to a nearby asymptotic state. Actually, as waves move along the duct, complicated wave interactions begin to occur; nevertheless, the above statements remain valid qualitatively.

Our basic estimate is on the change of speed of a shock wave as it moves along a duct. This and ohter estimates on wave interactions are established in Sect. 3. Our analysis is based on the random choice method of Liu [8], which generalizes the Glimm scheme, [3], for conservation laws. The idea is to decompose the solution into nonlinear modes for conservation laws and standing waves. In Sect. 4 we describe a variation of the simplified method of Liu [9]. Finally, in Sects. 5 and 6 , respectively, we study flows along a contracting duct and along an expanding duct.

\section{Preliminaries}

The gas dynamics equations (1.2) are supplemented by the constitutive relation

$$
p=p(v, s)
$$

where $s$ is the entropy of the gas, $v=1 / \varrho$ is the specific volume. These physical quantities satisfy the thermodynamics relation $d e=T d s-p d v, T$ the temperature of the gas. We assume that

$$
\partial p / \partial v<0 \text { and } \partial^{2} p / \partial v^{2}>0
$$

The characteristic speeds $\lambda_{i}, i=1,2,3$, and corresponding characteristic right vectors $r_{i}$ are [2]:

$$
\begin{aligned}
& \lambda_{1}=u-(-\partial p / \partial v)^{1 / 2} v, \quad \lambda_{2}=u, \quad \lambda_{3}=u+(-\partial p / \partial v)^{1 / 2}, \\
& \left.r_{1}=\left(1, \lambda_{1}, E+p v-u v(-\partial p / \partial v)^{1 / 2}\right)\right), \\
& \left.r_{3}=\left(1, \lambda_{3}, E+p v+u v(-\partial p / \partial v)^{1 / 2}\right)\right),
\end{aligned}
$$

and $r_{2}$ is such that $r_{2} \cdot \nabla u=r_{2} \cdot \nabla p=0$. Hereafter the coordinate system for the states is $\omega=(\varrho, \varrho u, \varrho E)$. The first inequality in (2.1) implies that $\lambda_{i}, i=1,2,3$, are real and distinct and so systems (1.1) and (1.2) are strictly hyperbolic. The second inequality in (2.1) implies that

$$
\begin{aligned}
& r_{1} \cdot \nabla \lambda_{1}=-1 / 2 \partial 2 p / \partial v^{2} \cdot(-\partial p / \partial v)^{-1 / 2} v^{3 / 2}<0 \\
& r_{3} \cdot \nabla \lambda_{3}=1 / 2 \partial 2 p / \partial v^{2} \cdot(-\partial p / \partial v)^{-1 / 2} v^{3 / 2}>0 .
\end{aligned}
$$


Direct calculations also yield:

$$
\begin{aligned}
& r_{1} \cdot \nabla u=-(-\partial p / \partial v)^{1 / 2} v^{2}<0, \quad r_{3} \cdot \nabla u=(-\partial p / \partial v)^{1 / 2} v^{2}>0, \\
& r_{1} \cdot \nabla p=r_{3} \cdot \nabla p=-v^{2} \partial p / \partial v>0 .
\end{aligned}
$$

We now describe the elementary waves for Euler equations (1.2). An $i$-rarefaction wave $\left(\omega_{0}, \omega_{1}\right), i=1,3$, is a smooth solution with speed $\lambda_{i}$ which depends on one parameter and connects $\omega_{0}$ on the left and $\omega_{1}$ on the right. It is a consequence of (1.2) that $\omega_{1}$ is on the $i$-rarefaction curve $R_{i}\left(\omega_{0}\right)$, the integral curve of $r_{i}$ through $\omega_{0}$, and $\lambda_{i}\left(\omega_{1}\right)>\lambda_{i}\left(\omega_{0}\right)$ :

$$
\omega_{1} \in R_{i}^{+}\left(\omega_{0}\right) \equiv\left\{\omega \in R_{i}\left(\omega_{0}\right), \lambda_{i}(\omega) \geqq \lambda_{i}\left(\omega_{0}\right)\right\} .
$$

There are two kinds of discontinuity waves. For the 2-characteristic field, $\left(\omega_{0}, \omega_{1}\right)$ is a discontinuity wave with speed $\sigma\left(\omega_{0}, \omega_{1}\right)$ provided

$$
\omega_{1} \in T_{2}\left(\omega_{0}\right) \equiv\left\{\omega: u(\omega)=u\left(\omega_{0}\right), p(\omega)=p\left(\omega_{0}\right)\right\}
$$

and is a constant discontinuity:

$$
\sigma\left(\omega_{0}, \omega_{1}\right)=\lambda_{2}\left(\omega_{0}\right)=\lambda_{2}\left(\omega_{1}\right)
$$

For the first and third characteristic fields, a discontinuity wave $\left(\omega_{0}, \omega_{1}\right)$ with speed $\sigma\left(\omega_{0}, \omega_{1}\right)$ satisfies the following jump (Rankine-Hugoniot) condition

$$
\begin{aligned}
\omega_{1} \in S_{1}^{-}\left(\omega_{0}\right) & \equiv\left\{\omega: \sigma\left(\omega-\omega_{0}\right)=f(\omega)-f\left(\omega_{0}\right)\right. \text { for some scalar, } \\
\sigma & \left.=\sigma\left(\omega_{0}, \omega\right): \lambda_{i}(\omega)<\lambda_{i}\left(\omega_{0}\right)\right\}, \quad i=1,3,
\end{aligned}
$$

and is a shock wave satisfying the following stability property, [1]:

$$
\lambda_{i}\left(\omega_{1}\right)<\sigma\left(\omega_{0}, \omega_{1}\right)<\lambda_{i}\left(\omega_{0}\right) .
$$

If we set

$$
T_{i}\left(\omega_{0}\right) \equiv S_{i}^{-}\left(\omega_{0}\right) \cup R_{i}^{+}\left(\omega_{0}\right), \quad i=1,3,
$$

then any state $\omega$ on $T_{i}\left(\omega_{0}\right)$ can be related to $\omega_{0}$ on the left by an $i$-elementary wave $\left(\omega_{0}, \omega\right)$. The curves $T_{1}\left(\omega_{0}\right)$ and $T_{3}\left(\omega_{0}\right)$ are $C^{2}$ at $\omega_{0}$ and monotone curves in the $(u, p)$-plane :

$$
\begin{array}{llll}
\left(p_{1}-p_{2}\right)\left(u_{1}-u_{2}\right)<0 & \text { for } & \omega_{1}, \omega_{2} \in T_{1}\left(\omega_{0}\right), & \omega_{1} \neq \omega_{2}, \\
\left(p_{1}-p_{2}\right)\left(u_{1}-u_{2}\right)>0 & \text { for } & \omega_{1}, \omega_{2} \in T_{3}\left(\omega_{0}\right), & \omega_{1} \neq \omega_{2} .
\end{array}
$$

The above holds in a small neighborhood of $\omega_{0}$ under the assumption (2.1). It holds globally under a stronger assumption, [5]:

$$
p=\bar{p}(v, e), \quad \partial \bar{p} / \partial v<0, \quad \partial \bar{p} / \partial e>0 .
$$

It follows from the thermodynamics relation $d e=T d s-p d v$ that $\partial \bar{p} / \partial e=T \partial p / \partial s$ and so the second inequality in (2.8) is equivalent to, [11]:

$$
p=p(v, s), \quad \partial p / \partial s>0 .
$$

To solve the Riemann problem (1.2) and (1.3) we find states $\omega_{m}$ and $\omega_{n}$ such that $\omega_{m} \in T_{1}\left(\omega_{\ell}\right), \omega_{n} \in T_{2}\left(\omega_{m}\right)$, and $\omega_{n} \in T_{3}\left(\omega_{r}\right)$ so that the solution consists of 1 -wave 
$\left(\omega_{\ell}, \omega_{m}\right), 2$-wave $\left(\omega_{m}, \omega_{n}\right)$, and 3-wave $\left(\omega_{n}, \omega_{r}\right)$. The states $\omega_{m}$ and $\omega_{n}$ are determined uniquely by $\omega_{\ell}$ and $\omega_{r}$ due to (2.7). We choose any nonsingular parameter $\tau_{i}$, $i=1,2,3$, along $T_{i}$ curves to measure the strength of waves:

$$
\begin{gathered}
\left(\omega_{\ell}, \omega_{n}\right)_{1} \equiv \tau_{1}\left(\omega_{\ell}\right)-\tau_{1}\left(\omega_{m}\right), \\
\left(\omega_{\ell}, \omega_{r}\right)_{2} \equiv \tau_{2}\left(\omega_{m}\right)-\tau_{2}\left(\omega_{n}\right), \\
\left(\omega_{\ell}, \omega_{r}\right)_{3} \equiv \tau_{3}\left(\omega_{n}\right)-\tau_{3}\left(\omega_{r}\right) .
\end{gathered}
$$

We choose $\tau_{i}$ so that shock waves have negative strength and rarefaction waves have positive strength.

\section{Basic Estimates}

The steady state equations (1.4) can also be written as

$$
\frac{d \omega}{d x}=1 / 2 c(x) \varrho u\left(\frac{r_{1}(\omega)}{\lambda_{1}(\omega)}+\frac{r_{3}(\omega)}{\lambda_{3}(\omega)}\right),
$$

where $c(x) \equiv-a^{\prime}(x) / a(x)$ which is assumed to be small in absolute value. In this section we will fix two space positions $x=x_{-}, x=x_{+}, x_{-}<x_{+}$, and call $\left(\omega_{0}, \omega_{1}\right)$ a standing wave if there exists a solution $\omega(x)$ of (1.4), or equivalently (3.1), such that $\omega\left(x_{-}\right)=\omega_{0}$ and $\omega\left(x_{+}\right)=\omega_{1}$. We will consider transonic flows with numerically positive speed, that is, $\left|\lambda_{1}(\omega)\right|$ is small. We first study the evolution of subsonic and supersonic waves as they propagate along the duct.

Lemma 3.1. Suppose that all the states are not sonic, that is, there exists $\lambda_{*}>0$ such that either $\lambda_{i}(\omega)>\lambda_{*}$ for all $\omega$ under consideration or $\lambda_{i}(\omega)<-\lambda_{*}$ for all $\omega$ under consideration. Let $\left(\omega_{\ell}, \bar{\omega}_{\ell}\right)$ and $\left(\omega_{r}, \bar{\omega}_{r}\right)$ be two standing waves. Then the strengths of elementary waves in the solutions of the Riemann problems $\left(\omega_{\ell}, \omega_{r}\right)$ and $\left(\bar{\omega}_{\ell}, \bar{\omega}_{r}\right)$ for (1.2) are related as follows:

$$
\begin{aligned}
\left(\omega_{\ell}, \omega_{r}\right)_{i} & =\left(\bar{\omega}_{\ell}, \bar{\omega}_{r}\right)_{i}+O(1) \alpha \bar{c}\left(\lambda_{*}\right)^{-2}, \quad i=1,2,3, \\
\alpha & \equiv\left\|\omega_{\ell}-\omega_{r}\right\|, \\
\bar{c} & \equiv \max _{x \leqq x \leqq x_{+}}|c(x)|,
\end{aligned}
$$

where $O(1)$ is a bound depending only on the system (1.2).

Proof. Since the waves $T_{i}, i=1,2,3$, are smooth, the solution of the Riemann problem depends continuously on the end states. Thus the lemma follows from the elementary theory of ordinary differential equations. We include $\left(\lambda_{*}\right)^{-2}$ on the right hand side of the estimate just to remind us that $\lambda_{*}$ may be a small number. Q.E.D.

The following crucial estimate is on the evolution of a 1-shock wave which is a perturbation of a standing 1-shock wave. It shows, in particular, that the shock wave accelerates as it propagates along a contracting duct and decelerates as it propagates along an expanding duct.

Lemma 3.2. Suppose that $\left(\omega_{\ell}, \omega_{r}\right)$ is a 1-shock wave which is a perturbation of a standing shock wave, that is, $\lambda_{1}\left(\omega_{\ell}\right)>0>\lambda_{1}\left(\omega_{r}\right)$ and its speed $\sigma \equiv \sigma\left(\omega_{\ell}, \omega_{r}\right)$ is small 
compared to its strength $\alpha \equiv\left|\left(\omega_{\ell}, \omega_{r}\right)\right|$. Suppose also that the duct is not uniform for $x \in\left(x_{-}, x_{+}\right)$, in other words, either $c / 2 \leqq c(x) \leqq c, x_{-}<x<x_{+}$or $c / 2 \leqq-c(x) \leqq c$, $x_{-} \leqq x \leqq x_{+}$, for some small positive constant $c$. Let $\left(\omega_{\ell}, \bar{\omega}_{\ell}\right)$ and $\left(\omega_{r}, \bar{\omega}_{r}\right)$ be two standing waves, then there exist $\omega_{*}$ on $S_{1}^{-}\left(\bar{\omega}_{\ell}\right)$ and a positive constant $d$ depending only on (1.2) such that

(i) $\left\|\omega_{*}-\bar{\omega}_{r}\right\|=O(1) c\left|x_{-}-x_{+}\right| \alpha^{2}$, and

(ii) when $c / 2 \leqq c(x) \leqq c$ for $x \in\left(x_{-}, x_{+}\right)$

$$
d c\left|x_{-}-x_{+}\right| \leqq \sigma\left(\bar{\omega}_{\ell}, \omega_{*}\right)-\sigma\left(\omega_{\ell}, \omega_{r}\right) \leqq 2 d c\left|x_{-}-x_{+}\right|,
$$

when $c / 2 \leqq-c(x) \leqq c$ for $x \in\left(x_{-}, x_{+}\right)$

$$
d c\left|x_{-}-x_{+}\right| \leqq \sigma\left(\omega_{\ell}, \omega_{r}\right)-\sigma\left(\bar{\omega}_{\ell}, \omega_{*}\right) \leqq 2 d c\left|x_{-}-x_{+}\right| .
$$

Proof. We will prove the lemma when $\sigma \geqq 0$ and $c / 2 \leqq c(x) \leqq c, x_{-}<x<x_{+}$; other cases are proved similarly. Let $\omega_{\ell}(x)$ and $\omega_{r}(x), x_{-}<x<x_{+}$, respectively, be the solution of (1.4) with $\omega_{\ell}\left(x_{+}\right)=\bar{\omega}_{\ell}, \omega_{\ell}\left(x_{-}\right)=\omega_{\ell}, \omega_{r}\left(x_{-}\right)=\omega_{r}$, and $\omega_{r}\left(x_{+}\right)=\bar{\omega}_{r}$. We will denote by $p_{t}(x)$ the pressure for the state $\omega_{t}(x)$ etc. Clearly we have $\lambda_{1}\left(\omega_{t}(x)\right)$ $>0>\lambda_{1}\left(\omega_{r}(x)\right), x_{-} \leqq x \leqq x_{+}$. We have by integrating (1.4)

$$
\begin{aligned}
\bar{\varrho}_{\ell} \bar{u}_{\ell} & =K \varrho_{\ell} u_{\ell}, \quad \bar{\varrho}_{r} \bar{u}_{r}=K \varrho_{r} u_{r}, \\
\varrho_{\ell} \bar{u}_{\ell}^{2}+\bar{p}_{\ell} & =K\left(\varrho_{\ell} u_{\ell}^{2}+p_{\ell}\right)+\int_{x_{-}}^{x_{+}} K(y) c(y) p_{\ell}(y) d y, \\
\bar{\varrho}_{r} \bar{u}_{r}^{2}+\bar{p}_{r} & =K\left(\varrho_{r} u_{r}^{2}+p_{r}\right)+\int_{x_{-}}^{x_{+}} K(y) c(y) p_{r}(y) d y, \\
\bar{\varrho}_{\ell} \bar{E}_{\ell} \bar{u}_{\ell}+\bar{p}_{\ell} \bar{u}_{\ell} & =K\left(\varrho_{\ell} E_{\ell} u_{\ell}+p_{\ell} u_{\ell}\right), \\
\bar{\varrho}_{r} \bar{E}_{r} \bar{u}_{r}+\bar{p}_{r} \bar{u}_{r} & =K\left(\varrho_{r} E_{r} u_{r}+p_{r} u_{r}\right), \\
K(y) & \equiv \exp \left[-\int_{x-}^{y} c(z) d z\right] \\
K & \equiv K\left(x_{+}\right) .
\end{aligned}
$$

We choose $\bar{\omega}$ on $S_{1}^{-}\left(\bar{\omega}_{\ell}\right)$ such that

$$
\sigma\left(\bar{\omega}_{\ell}, \bar{\omega}\right)=\sigma\left(\omega_{\ell}, \omega_{r}\right) \equiv \sigma .
$$

It follows from (3.3) and the jump condition for $\left(\omega_{\ell}, \omega_{r}\right)$ and $\left(\bar{\omega}_{\ell}, \bar{\omega}\right)$ that

$$
\begin{aligned}
\sigma\left(\varrho_{\ell}-\varrho_{r}\right) & =\varrho_{\ell} u_{\ell}-\varrho_{r} u_{r}, \quad \sigma\left(\bar{\varrho}_{\ell}-\bar{\varrho}\right)=\bar{\varrho}_{\ell} \bar{u}_{\ell}-\overline{\varrho u}, \\
\sigma\left(\varrho_{\ell} u_{\ell}-\varrho_{r} u_{r}\right) & =\left(\varrho_{\ell} u_{\ell}^{2}+p_{\ell}\right)-\left(\varrho_{r} u_{r}^{2}+p_{r}\right), \\
\sigma\left(\varrho_{\ell} \bar{u}_{\ell}-\overline{\varrho u}\right) & =\left(\varrho_{\ell} \bar{u}_{\ell}^{2}+\bar{p}_{\ell}\right)-\left(\overline{\varrho u}^{2}+\bar{p}\right), \\
\sigma\left(\varrho_{\ell} E_{\ell}-\varrho_{r} E_{r}\right) & =\left(\varrho_{\ell} E_{\ell} u_{\ell}+p_{\ell} u_{\ell}\right)-\left(\varrho_{r} E_{r} u_{r}+p_{r} u_{r}\right), \\
\sigma\left(\varrho_{\ell} \bar{E}_{\ell}-\overline{\varrho E}\right) & =\left(\varrho_{\ell} \bar{E}_{\ell} \bar{u}_{\ell}+\bar{p}_{\ell} \bar{u}_{\ell}\right)-(\overline{\varrho E u}+\overline{p u}) .
\end{aligned}
$$

We have from (3.2) ${ }_{1}$ and the first identity in $(3.4)_{1}$, that

$$
\sigma K\left(\varrho_{\ell}-\varrho_{r}\right)=\bar{\varrho}_{\ell} \bar{u}_{\ell}-\bar{\varrho}_{r} \bar{u}_{r} .
$$


This, and the second identity in $(3.4)_{2}$ yield

$$
\bar{\varrho}_{r} \bar{u}_{r}-\varrho u=\sigma\left[\left(\bar{\varrho}_{\ell}-\bar{\varrho}\right)-K\left(\varrho_{\ell}-\varrho_{r}\right)\right] \text {. }
$$

We have from $(3.2)_{2}$ and the first identity in $(3.4)_{2}$ that

$$
\left(\bar{\varrho}_{r} \bar{u}_{r}^{2}+\bar{p}_{r}\right)-\left(\bar{\varrho}_{\ell} \bar{u}_{\ell}^{2}+\bar{p}_{\ell}\right)=\sigma\left(\varrho_{r} u_{r}-\varrho_{\ell} u_{\ell}\right)+\int_{x_{-}}^{x_{+}} K(y) c(y)\left(p_{r}(y)-p_{\ell}(y)\right) d y .
$$

This, (3.2) $)_{1}$, and the second identity in $(3.4)_{2}$ yield

$$
\left(\bar{\varrho}_{r} \bar{u}_{r}^{2}+\bar{\varrho}_{r}\right)-\left(\overline{\varrho u}^{2}+\bar{p}\right)=\sigma\left(\bar{\varrho}_{r} \bar{u}_{r}-\overline{\varrho u}\right)+\int_{x_{-}}^{x_{+}} K(y) c(y)\left[p_{r}(y)-p_{\ell}(y)\right] d y .
$$

From $(3.4)_{1}$, we have

$$
\varrho_{\ell}\left(\sigma-u_{\ell}\right)=\varrho_{r}\left(\sigma-u_{r}\right) \equiv A, \quad \varrho_{\ell}\left(\sigma-\bar{u}_{\ell}\right)=\bar{\varrho}(\sigma-\bar{u}) \equiv B
$$

whence we have from $(3.4)_{3}$ that

$$
\begin{aligned}
& E_{\ell}=E_{r}+\left(p_{\ell} u_{\ell}-p_{r} u_{r}\right) / A, \\
& \bar{E}_{\ell}=\bar{E}+\left(\bar{p}_{\ell} \bar{u}_{\ell}-\overline{p u}\right) / B .
\end{aligned}
$$

$(3.2)_{1}$ and $(3.2)_{3}$ imply

$$
\begin{aligned}
& \bar{E}_{r}+\bar{p}_{r} / \bar{\varrho}_{r}=E_{r}+p_{r} / \varrho_{r} \\
& \bar{E}_{\ell}+\bar{p}_{\ell} / \bar{\varrho}_{\ell}=E_{\ell}+p_{\ell} / \varrho_{\ell} .
\end{aligned}
$$

Thus we have from the above four identities that

$$
\begin{aligned}
\left(\bar{E}_{r}+\right. & \left.\bar{p}_{r} / \bar{\varrho}_{r}\right)-(\bar{E}-\bar{p} / \varrho) \\
= & E_{r}+p_{r} / \varrho_{r}-\bar{E}_{\ell}+\left(\bar{\varrho}_{\ell} \bar{u}_{\ell}-\overline{\varrho u}\right) / B+\bar{p} / \varrho \\
= & E_{\ell}-\left(\varrho_{\ell} u_{\ell}-p_{r} u_{r}\right) / A+p_{r} / \varrho_{r}-E_{\ell}-p_{\ell} / \varrho_{\ell}+\bar{p}_{\ell} / \varrho_{\ell} \\
& +\left(\bar{\varrho}_{\ell} \bar{u}_{\ell}-\overline{\varrho u}\right) / B+\bar{p} / \varrho,
\end{aligned}
$$

which can be simplified to

$$
\left(\bar{E}_{\ell}+\bar{p}_{r} / \bar{\varrho}_{r}\right)-(\bar{E}-\bar{p} / \bar{\varrho})=\sigma\left[\frac{\bar{p}-\bar{p}_{\ell}}{\bar{\varrho}_{\ell}\left(\bar{u}_{\ell}-\sigma\right)}-\frac{p_{r}-p_{\ell}}{\varrho_{\ell}\left(u_{\ell}-\sigma\right)}\right] .
$$

We now finish the proof of the lemma based on $(3.5)_{1},(3.5)_{2}$, and $(3.5)_{3}$. We have from $(3.5)_{1}$ that

$$
\begin{aligned}
K & =1+O(1) c\left|x_{+}-x_{-}\right| \\
\varrho_{r} \bar{u}_{r}-\overline{\varrho u} & =\sigma\left\{\left(\varrho_{\ell}-\varrho_{\ell}\right)+\left(\varrho_{r}-\varrho\right)\right\}+\sigma(1-K)\left(\varrho_{1}-\varrho_{2}\right) .
\end{aligned}
$$

On the other hand we have from (3.1) that

$$
\begin{aligned}
\left|\bar{\varrho}_{\ell}-\varrho_{\ell}\right| & =O(1) c\left(\lambda_{*}\right)^{-1}\left|x_{+}-x_{-}\right|, \\
\left|\varrho_{r}-\bar{\varrho}\right| & =O(1) c\left(\lambda_{*}\right)^{-1}\left|x_{+}-x_{-}\right|,
\end{aligned}
$$


where $\lambda_{*}$ is the minimum of $\left|\lambda_{i}(\omega)\right|$ for all $\omega$ under consideration. Since $\sigma$ and $|c(x)|$ are small compared to the strength $\alpha$ of the shock wave $\left(\omega_{\ell}, \omega_{r}\right)$, we know that $\lambda_{*}$ is of the same order of $\alpha$ and it follows from the last four estimates that

$$
\begin{aligned}
\bar{\varrho}_{r} \bar{u}_{r}-\overline{\varrho u} & =O(1) \sigma c\left|x_{+}-x_{-}\right|\left(\alpha^{-1}+\alpha\right) \\
& =O(1) \sigma c\left|x_{+}-x_{-}\right| \alpha^{-1} .
\end{aligned}
$$

Note also that $p_{r}(y)-p_{t}(y), x_{-} \leqq y \leqq x_{+}$, is of the same order as $\alpha$ and so $(3.6)_{1}$ and $(3.5)_{2}$ yield

$$
\begin{aligned}
& 2 c \alpha\left|x_{-}-x_{+}\right|+O(1) \sigma^{2} c\left|x_{+}-x_{-}\right| \alpha^{-1} \\
& \quad \geqq\left(\bar{\varrho}_{r} \bar{u}_{r}^{2}+\bar{p}_{r}\right)-\left(\varrho \bar{\varrho}^{2}+\bar{p}\right) \geqq 1 / 3 c \alpha\left|x_{-}-x_{+}\right|+O(1) \sigma^{2} c\left|x_{+}-x_{-}\right| \alpha^{-1},
\end{aligned}
$$

where we have defined the strength $\alpha$ by the difference in $p$ just for convenience. Since $\sigma$ is small compared to $\alpha$ the above estimate becomes

$$
\begin{aligned}
3 c \alpha\left|x_{-}-x_{+}\right| & \geqq\left(\bar{\varrho}_{r} \bar{u}_{r}^{2}+\bar{p}_{r}\right)-\left(\overline{\varrho u}^{2}+\bar{p}\right) \\
& \geqq 1 / 4 c \alpha\left|x_{-}-x_{+}\right| .
\end{aligned}
$$

Similarly we have from $(3.5)_{3}$ that

$$
\left(\bar{E}_{r}+\frac{\bar{p}_{r}}{\bar{\varrho}_{r}}\right)-\left(\bar{E}+\frac{\bar{p}}{\bar{\varrho}}\right)=O(1) c \sigma\left|x_{-}-x_{+}\right| \alpha^{-1} .
$$

Let us denote by $\Gamma$ any curve which is the interaction of surfaces $\varrho u=$ const and $E+p / \varrho=$ const. The tangent $T_{\Gamma}$ to $\Gamma$ is the cross product of $n_{1} \equiv \nabla \varrho u$ and $n_{2} \equiv \nabla(E+p / \varrho)$. Direct calculations yield

$$
\begin{gathered}
T_{\Gamma}=r_{1}+\lambda_{1}\left(0,-1,-u-\lambda_{1}\left(1+\bar{\varrho}_{e} v\right)\right), \\
n_{1} \cdot n_{2}=-\bar{\varrho}_{e} u v^{2} \neq 0, \\
T_{\Gamma} \cdot \nabla\left(\varrho u^{2}+p\right)=-\frac{\lambda_{1} \lambda_{3}}{1+\bar{\varrho}_{e} v} .
\end{gathered}
$$

Estimates $(3.6)_{1},(3.6)_{2}$, and (3.8) imply that there exists $\tilde{\omega}$ on $\Gamma(\bar{\omega})$ such that

$$
\left\|\tilde{\omega}-\bar{\omega}_{r}\right\|=O(1) c \sigma\left|x_{-}-x_{+}\right| \alpha^{-1} .
$$

From our hypothesis we know that $\sigma \alpha^{-1}$ is small compared to $\alpha$ and $\left|\lambda_{1}(\omega)\right|$ is of the same order as $\alpha$ for all $\omega$ under consideration. Thus we have from (3.10) that

$$
\tilde{\omega}-\bar{\omega}=\bar{\omega}_{r}-\bar{\omega}+O(1) c \sigma\left|x-x_{+}\right| \alpha^{-1},
$$

and so (3.9) yields

$$
\begin{aligned}
d_{1} \alpha^{-1}\left|\left(\bar{\varrho}_{r} \bar{u}_{r}^{2}+\bar{p}_{r}\right)-\left(\overline{\varrho u}^{2}+\bar{p}\right)\right| & \leqq\|\tilde{\omega}-\bar{\omega}\|+O(1) c \sigma\left|x_{-}-x_{+}\right| \alpha^{-1} \\
& \leqq 2 d_{1} \alpha^{-1}\left|\left(\bar{\varrho}_{r} \bar{y}_{r}^{2}+\bar{p}_{r}\right)-\left(\overline{\varrho u}^{2}+\bar{p}\right)\right|
\end{aligned}
$$

for some positive constant $d_{1}$ depending only on (1.2). The above estimate and (3.6) $)_{2}$ imply that for some positive constant $d_{0}$

$$
0<d_{0} \leqq\|\tilde{\omega}-\bar{\omega}\|\left(c\left|x_{-}-x_{+}\right|\right)^{-1} \leqq 2 d_{0}<\infty .
$$


It is clear from (2.6) and (3.7) that

$$
T_{\Gamma} \cdot \nabla p>0
$$

and so (3.9), (3.6) 2 , and (3.11) imply that

$$
\bar{p}_{r}-\bar{p}>0 \text {. }
$$

It follows from (3.7), (3.10), and (3.11) that there exists $\hat{\omega}$ on $R_{1}(\bar{\omega})$ such that

$$
\begin{aligned}
&\left\|\hat{\omega}-\bar{\omega}_{r}\right\| \leqq\|\tilde{\omega}-\bar{\omega}\|+\left\|\tilde{\omega}-\bar{\omega}_{r}\right\| \\
&=O(1) c\left|x_{-} x_{+}\right|\left\{\sigma \alpha^{-1}+\alpha^{2}\right\}, \\
& 0<d_{0} \leqq\|\hat{\omega}-\bar{\omega}\|\left(c\left|x_{-}-x_{+}\right|\right)^{-1} \leqq 2 d_{0}<\infty, \\
& \hat{p}-\bar{p}>0 .
\end{aligned}
$$

It is well known that $S_{i}\left(\omega_{0}\right)$ is tangent to $R_{i}\left(\omega_{0}\right)$ up to second order (cf. [2]). Since $\bar{\omega}$ is on $S_{1}^{-}\left(\bar{\omega}_{\ell}\right)$, there exists $\omega_{*}$ on $S_{i}^{-}\left(\bar{\omega}_{\ell}\right)$ such that

$$
\left\|\omega_{*}-\hat{\omega}\right\|=O(1) \alpha^{2}\|\bar{\omega}-\hat{\omega}\|,
$$

which and $(3.12)_{1}$ yield

$$
\begin{aligned}
\left\|\bar{\omega}_{r}-\omega_{*}\right\| & \leqq\left\|\bar{\omega}_{r}-\hat{\omega}\right\|+\left\|\hat{\omega}-\omega_{*}\right\| \\
& =O(1) c\left|x_{-}-x_{+}\right|\left\{\sigma \alpha^{-1}+\alpha^{1}+\alpha^{2}\right\} \\
& =O(1) x\left|x_{-}-x_{+}\right| \alpha^{2} .
\end{aligned}
$$

This proves (i) of the lemma; (ii) follows from $(3.12)_{2}$ and $(3.12)_{3}$. Q.E.D.

\section{Numerical Schemes}

Since we will be dealing with instability phenomena, it is important that numerical schemes used to calculate the solution are effective so that approximate solutions do not oscillate around the unstable solution or bifurcate into different branches of stable solutions. We will describe two variations of the random choice scheme which was introduced in [8], simplified in [9] and is a generalization of the Glimm scheme for conservation laws, [3,7]. For convenience we write (1.1) as

$$
\frac{\partial \omega}{\partial t}+\frac{\partial f(\omega)}{\partial x}=g(x, \omega)
$$

and the initial data, Euler equations and steady state equations become $\omega(x, 0)=\omega_{0}(x)$

$$
\begin{aligned}
& \frac{\partial \omega}{\partial t}+\frac{\partial f(\omega)}{\partial x}=0 \\
& \frac{\partial f(\omega)}{\partial x}=g(x, \omega) .
\end{aligned}
$$

We now describe the first scheme for calculating the solution of the initial value problem for (1.1). Choose an equidistributed sequence $\left\{a_{k}\right\}_{k=0}^{\infty}$ in $(0,1)$, and mesh 
length $\Delta x=r, \Delta t=s$ satisfying the usual Courant-Friedrichs-Lewy condition. The approximate solution $\omega_{r}(x, t) \equiv \omega_{r}\left(x, t ;\left\{a_{k}\right\}\right)$ is defined inductively as follows: Suppose that $\omega_{r}(x, t)$ has been defined for $0 \leqq t \leqq k s, k$ a nonnegative integer. Then $u_{r}(x, k s+0)$ is defined according to $a_{k}$ :

$$
\begin{gathered}
\omega_{r}\left(h r+a_{k} r, k s+0\right) \equiv \omega_{r}\left(h r+a_{k} r, k s\right), h \text { integer } \\
\omega_{r}(x, k s+0) \text { is a solution for }(1.4),(h-1) r<x<h r, h \text { integer } .
\end{gathered}
$$

Thus $\omega_{r}(x, k s+0)$ consists of standing waves with possible discontinuities at $x=h r$, $h$ integer. We now resolve these discontinuities so that $\omega_{r}(x, t)$ is defined for $k s<0$ $\leqq(k+1) s$. To resolve the discontinuity, for example, at $x=0$, we first solve the Riemann problem for (1.2) with

$$
\omega(x, 0)= \begin{cases}\omega_{r}(x-0, k s+0) & x<0 \\ \omega_{r}(x+0, k s+0) & x<0\end{cases}
$$

and denote the solution by $\omega_{*}(x, t)=\psi(x / t)$. Then we perturb $\psi(x / t)$ by (1.4) to obtain an approximate solution to (1.1):

and

$$
\bar{\omega}(x, t) \equiv \psi(\xi) \quad \text { when } \quad \xi=x / t \quad \text { and } \quad t \rightarrow 0
$$

$$
\bar{\omega}(x, x / \xi) \text { satisfies }(1.4) \text {. }
$$

Note that $\bar{\omega}(x, t)$ exists locally in time and is uniquely determined by $\omega_{r}(x, k s)$ provided that (1.4) can be solved locally, which is the case if $\lambda_{i}(\omega), i=1,3$, is nonzero for all states $\omega$ under consideration. Finally, near $(0, k s) \omega_{r}(x, t)$ is defined as a translation of $\bar{w}(x, t): \omega_{r}(x, t-k s)=\bar{w}(x, t)$. The discontinuities $(h r, k s)$ in $\omega_{r}(x, k s)$ are resolved in a similar way. This defines the approximate solution $\omega_{r}(x, t)$ for all $(x, t)$. Note that in addition to solving the Riemann problem, which is needed in the Glimm scheme for conservation laws, we only have to solve the ordinary differential equation (1.4) at most once for each time and space step. When $a_{k} r / s$ is between the maximum (minimum) speed of waves issued from $(h r,(k-1) s), \quad(((h+1) r,(k-1) s)) \quad$ then $\quad \omega_{r}(x,(k+1) s-0)=\omega_{r}(x,(k+1) s+0)$ for $h r<x<(h+1) r$ and we don't even have to solve (1.4). When $a_{k} r / s$ is, say, less than the maximum speed of the waves issued from $(h r, k s)$, then $\omega_{r}(h r+0,(k+1) s+0)$ is the same as $\psi\left(a_{k} r / s\right)$ where $\psi$ is the solution of the corresponding Riemann problem at $(h r, k s)$. However one has to solve (1.4) with $\omega_{r}(h r+0,(k+1) s+0)$ as given to find the value of $\omega_{r}((h+1) r-0,(k+1) s)$.

The above scheme, when system (1.1) is reduced to conservation laws (1.3), does not stagger the grid points $(h r, k s)$ as was done originally in Glimm [3]. This makes the scheme numerically more efficient. For the present study it is particularly convenient, since waves with positive (negative) speed are never moved to the left (right). As a consequence the scheme would approximate the asymptotic state of an unstable solution accurately, (see next Section). When one is interested in flows with a single relatively strong shock wave the following variation has the advantage in tracing the evolution of the shock wave. Suppose that at time $t=k s$, the position of the shock wave is $x=x_{k},(h-1) r \leqq x_{k}<h r$. Then we disregard the grid points $((h-1) r, k s)$ and $(h r, k s)$ and extend the standing wave 
in $\omega_{r}(x, k s+0)$ for $h r<x<(h+1) r$ [or $\left.(h-2) r<x<(h-1) r\right]$ to the interval $x_{k}<x<h r\left[\right.$ or $\left.(h-1) r<x<x_{k}\right]$ so that $\omega_{r}(x, k s+0)$ is discontinuous at $x=x_{k}$ and continuous at $x=(h-1) r$ and $x=h r$. This has the advantage that the position of the shock wave is a continuous function of time. In the present study we will use the first scheme just for simplicity in the presentation.

The existence theory is based on the estimates on the total variation of the approximation solution. This is usually proved by induction. For a later purpose we now introduce some of the notations. An $I$-curve $J$ is a spacelike curve consisting of lines between $\left.\left(h+a_{k}\right) r, k s\right)$ and $(h r,(k+1) s)$ or between $\left(\left(h+a_{k}\right), k s\right)$ and $((h+1) r,(k \pm 1) s), h$ any integer, $k$ any positive integer. $J_{2}$ is called an immediate successor of $J_{1}$ if $J_{2}$ lies toward a larger time than $J_{1}$ and they pass through some grid points except one. The region between $J_{1}$ and $J_{2}$ is called a diamond $\Delta$. The strength of waves issued from $(h r, k s)$ is measured by its initial strength at $(h r, k s)$. Suppose $\Delta$ is centered at $(h r,(k+1) s)$. Then waves entering $\Delta$, besides those issued from $(h r, k s)$, come either from $((h-1) r, k s)$ or $((h+1) r, k s)$. The strength of waves issued from $(h r, k s)$ is unchanged at $(h r,(k+1) s-0)$. Those issued from $((h-1) r, k s)$ [or $((h+1) r, k s)]$ have positive (or negative) speed and the difference of their strength between times $k s+0$ and $(k+1) s-0$ is due to the geemetry of the duct between $x=(h-1) r$ and $x=h r$ [or between $x=h r$ and $x=(h+1) r$ ] and is estimated by Lemma 3.1. The waves leaving $\Delta$ are then the result of nonlinear interaction for (1.2) of waves inside $\Delta$ at time $(k+1) s-0$ just mentioned. Such interactions have been studied in $[3,6]$.

The scheme just introduced converges and yields an exact solution provided that no state is near sonic and the total variation of the approximation solution is uniformly bounded $[8,9]$.

\section{Unstable Flows}

In this section we study flows with positive speed along a contracting duct. For definiteness we assume:

$$
\begin{array}{ll}
c(x) \equiv-\frac{a^{\prime}(x)}{a(x)}>0 & \text { for } 0<x<1 \\
c(x) \equiv 0 & \text { otherwise }
\end{array}
$$

Consider the solution $\omega_{*}(x, t)=\omega_{*}(x)$ of $(1.1)$ which consists of standing waves and a standing shock wave at $x=x_{*}, 0 \leqq x_{*} \leqq 1$ :

$$
\omega_{*}(x, t)= \begin{cases}\omega_{1}(x) & 0 \leqq x<x_{*} \\ \omega_{2}(x) & x_{*}<x \leqq 1 \\ \omega(-\infty) & x<0 \\ \omega(+\infty) & x>1,\end{cases}
$$

where $\omega_{1}(x)$ and $\omega_{2}(x)$ are solutions of $(1.4),\left(\omega_{1}\left(x_{*}\right), \omega_{2}\left(x_{*}\right)\right)$ is a 1-shock wave with

$$
\sigma\left(\omega_{1}\left(x_{*}\right), \omega_{2}\left(x_{*}\right)\right)=0 \text {, }
$$


and $\omega(-\infty) \equiv \omega_{1}(0), \omega(+\infty) \equiv \omega_{2}(1)$. Before we study the instability of standing shock waves, we first show that wave patterns of other kinds are stable. We will then show that a small perturbation of a standing shock wave causes the unstable flow to approach one of the stable flows. By a noninteracting wave pattern we mean a solution of (1.1) consisting of elementary waves for Euler equations (1.3) in the region $x \notin(0,1)$ and of standing waves and a possible standing shock wave in $x \in(0,1)$. Moreover, these waves do not interact in the positive time direction, in other words, they do not occupy the same space position for any positive time.

Theorem 5.1. Suppose that $\bar{\omega}(x, t)$ is a noninteracting wave pattern for (1.1) with the property that $\bar{\omega}(x, t)$ does not contain any standing shock wave and all waves in $\bar{\omega}(x, t)$ are weak and $\lambda_{1}(\omega) \neq 0$ for $\omega=\bar{\omega}(x, t)$. Then $\bar{\omega}(x, t)$ is asymptotically stable in the sense that if an initial data $\omega(x, 0)$ is such that the total variation of $\omega(x, 0)-\bar{\omega}(x, 0)$ is small, then the solution $\omega(x, t)$ of $(1.1)$ tends to an asymptotic state consisting of elementary waves which are close to those in $\bar{\omega}(x, t)$ both in position and strength.

Proof. We may let the perturbation $\omega(x, 0)-\bar{\omega}(x, 0)$ be so small that $\omega(x, t)$ is never sonic and so the schemes described in the previous sections may be applied. Note that since $\bar{\omega}(x, t)$ contains no standing shock wave, the standing wave in it is either strictly supersonic or strictly subsonic. Although Liu [8] studies only flows which are supersonic (or subsonic) throughout the duct, the ideas employed there can be generalized to prove the present theorem. We omit the details. Q.E.D.

Theorem 5.2. The wave pattern $\omega_{*}(x, t),(5.2)$ and (5.3), is dynamically unstable provided that $|c(x)|$ is small as compared to the strength $\alpha_{0}$ of the standing wave $\left(\omega_{1}\left(x_{*}\right), \omega_{2}\left(x_{*}\right)\right)$. More precisely, there exists $\omega(x, 0)$ such that the total variation of $\omega(x, 0)-\omega_{*}(x, 0)$ is arbitrarily small and as time goes to infinity $\omega(x, t)$ tends to one of the stable asymptotic states without a standing shock wave as studied in the previous theorem.

Proof. For definiteness we assume that $0 \leqq x_{*}<1$. We choose the perturbation $\omega(x, 0)$ to consist of standing waves and a 1 -shock wave at $x=x_{*}$ with positive speed. This can be done quite easily by choosing a state $\bar{\omega}$ on $S_{1}^{-}\left(\omega_{1}\left(x_{*}\right)\right)$ which is between $\omega_{1}\left(x_{*}\right)$ and $\omega_{2}\left(x_{*}\right)$ and arbitrarily close to $\omega_{2}\left(x_{*}\right)$, and define $\omega(x, 0)$ by:

$$
\omega(x, 0) \equiv \begin{cases}\omega_{1}(x), & 0 \leqq x<x_{*}, \\ \bar{\omega}_{2}(x), & x_{*}<x \leqq 1 \\ \omega_{*}(x, t) & \text { otherwise },\end{cases}
$$

where $\bar{\omega}_{2}(x)$ is a standing wave with $\bar{\omega}_{2}\left(x_{*}\right)=\bar{\omega} . \omega(x, 0)$ is a small perturbation of $\omega_{*}(x, t)$ since $\bar{\omega}$ is close to $\omega_{2}\left(x_{*}\right)$. Moreover we have

$$
\sigma\left(\omega\left(x_{*}-0,0\right), \omega\left(x_{*}+0,0\right)\right)>0 .
$$

For convenience we choose $\Delta x=r$ such that $\left(x_{*}, 0\right)$ is a grid point. We will use the first scheme described in the previous section. We first establish the existence of the solution $\omega(x, t)$. The first step is to establish a uniform bound on the total variation of the approximate solution $w_{2}(x, t)$. We will show inductively that there is a relatively strong 1 -shock wave in $w_{2}(x, t)$ which originates at $\left(x_{*}, 0\right)$ and persists for 
all time. Suppose that it crosses an $I$-curve $J$ at $x=h_{0} r$ and has strength $\alpha_{*}$. Then we define a nonlinear functional $F(J)$ as follows:

$F(J) \equiv L(J)+K\left\{Q_{1}(J)+Q_{2}(J)+Q_{3}(J)+Q_{d}(J)\right\}$,

$L(J) \equiv \sum\{|\alpha|: \alpha$ the strength of any elementary wave crossing $J\}$,

$Q_{1}(J) \equiv Q_{1}^{\ell}(J)+Q_{1}^{r}(J)+Q_{1}^{c}(J)+Q_{1}^{s}(J)$,

$Q_{1}^{\ell}(J) \equiv \sum\left\{|\alpha| \int_{h r}^{\infty}|c(x)| d x: \alpha\right.$ is the strength of a 1 -wave which crosses $J$ issues from $\left.(h r, k s), h \leqq h_{0}\right\}$

$Q_{1}^{r}(J) \equiv \sum\left\{|\alpha| \int_{h_{0} r}^{\infty}|c(x)| d x+|\alpha| \int_{h_{0} r}^{h r}|c(x)| d x: \alpha\right.$ is the strength of a 1-wave which crosses $Q_{1}^{c} \equiv \alpha_{*} \int_{h_{0} r}^{\infty}|c(x)| d x$,

$J$ issues from $\left.(h r, k s), h \geqq h_{0}\right\}$,

$Q_{1}^{s}(J) \equiv \sum\{|\alpha \beta|: \alpha$ and $\beta$ are strengths of 1 -wave which crosses $J$ and at least one of them is a shock wave ,

$Q_{2}(J) \equiv \sum\left\{|\alpha| \int_{h r}^{\infty}: \alpha\right.$ is the strength of a 2 -wave which crosses $J$ issues from $\left.(h r, k s)\right\}$, $Q_{3}(J) \equiv Q_{3}^{\prime}(J)+Q_{3}^{s}(J)$

$Q_{3}^{\prime}(J) \equiv \sum\left\{|\alpha| \int_{h r}^{\infty}|c(x)| d x: \alpha\right.$ is a strength of a 3 -wave which crosses $J$ issues from $(h r, k s)\}$,

$Q_{3}^{s}(J) \equiv \sum\{|\alpha \beta|: \alpha$ and $\beta$ are strengths of two 3-wave which crosses $J$ and at least one of them is a shock wave $\}$,

$Q_{d}(J) \equiv \sum\{|\alpha \beta|: \alpha$ and $\beta$, respectively, are strengths of a $i$-wave to the right a $j$-wave to the left crossing $J$ and $i<j\}$,

where $K$ is some large number to be determined later.

The terms $Q$ 's are defined so as to detect the potential amount of wave interactions in the solution. For instance, $Q_{1}^{c}$ is so defined as to anticipate that the relatively strong shock wave would move to the right, (cf. Lemma 4.2). $Q_{1}^{\ell}$ and $Q_{1}^{r}$ are defined to reflect the fact that 1 -waves on either side of the relatively strong 1 -shock wave move toward it and are eventually combined into the right moving relatively strong shock wave. This is the case if the shock wave issued from $\left(x_{*}, 0\right)$ remains relatively strong for all time and always has positive speed. This, and other qualitative properties, will be proved simultaneously as we establish the bound on the total variation of $w_{r}$ below.

We will prove by induction that for any $I$-curve $J$

$$
\begin{aligned}
F(J) & \leqq F(0), \\
Q(\Lambda) & \leqq 2[Q(0)-Q(J)],
\end{aligned}
$$


where $\Lambda$ is the region below $J$ and 0 is the (unique) $I$-curve in the zone $0 \leqq t \leqq s$. The term $\Lambda$ is defined to be the sum of all $Q(\Delta), \Delta$ any diamond in $A$, and $Q(\Delta)$ is defined as follows: Suppose that waves entering $\Delta$ are solutions of the Riemann problems $\left(\omega_{1}, \omega_{2}\right)$ and $\left(\omega_{3}, \omega_{4}\right)$ and are centered at $\left(h_{1} r, k s\right)$ and $\left(h_{2} r, k s\right), h_{1}=h_{2}-1$, respectively. Thus the center of $\Delta$ is $(h r,(k+1) s), h$ equal to $h_{1}$ or $h_{2}$. When $h$ equals $h_{1}$, the waves leaving $\Delta$ is the solution of the Riemann problem $\left(\omega_{1}, \bar{\omega}_{4}\right)$ and is the result of the interaction of waves in the Riemann problems $\left(\omega_{1}, \omega_{2}\right)$ and $\left(\bar{\omega}_{3}, \bar{\omega}_{4}\right)$. Here $\left(\bar{\omega}_{3}, \omega_{3}\right)$ and $\left(\bar{\omega}_{4}, \omega_{4}\right)$ are standing waves in the interval $x \in(h r,(h+1) r)$. Note that in this case waves in $\left(\omega_{3}, \omega_{4}\right)$ have negative speed and move from $x=(h+1) r$ to $x=h r$ during the time $t \in(k s,(k+1) s]$. We set (cf. [8])

$$
\begin{aligned}
Q(\Delta) \equiv & \left(\int_{h r}^{(h+1)}|c(x)| d x\right) \cdot \sum_{i}\left|\left(\omega_{3}, \omega_{4}\right)_{i}\right|+\sum_{i>j}\left|\left(\omega_{1}, \omega_{2}\right)_{i}\left(\omega_{3}, \omega_{4}\right)_{j}\right| \\
& +\sum_{i}\left\{\left|\left(\omega_{1}, \omega_{2}\right)_{i}\left(\omega_{3}, \omega_{4}\right)_{i}\right|: \text { at least one of }\left(\omega_{1}, \omega_{2}\right)\right. \\
& \left.\quad \text { and }\left(\omega_{3}, \omega_{4}\right)_{i} \text { is a shock wave }\right\}
\end{aligned}
$$

Similarly, when $h=h_{2}$, waves in $\left(\omega_{1}, \omega_{2}\right)$ have positive speed and move from $x=(h-1) r$ to $x=h r$ during the time $t \in(k s,(k+1) s]$. We set

$$
\begin{aligned}
Q(\Delta) \equiv & \left(\int_{(h-1) r}^{h r}|c(x)| d x\right) \cdot \sum_{i}\left|\left(\omega_{1}, \omega_{2}\right)_{i}\right|+\sum_{i>j}\left|\left(\omega_{1}, \omega_{2}\right)_{i}\left(\omega_{3}, \omega_{4}\right)_{j}\right| \\
& +\sum_{i}\left\{\left|\left(\omega_{1} \cdot \omega_{2}\right)_{i}\left(\omega_{3}, \omega_{4}\right)_{i}\right|: \text { at least one of }\left(\omega_{1}, \omega_{2}\right)_{i}\right. \\
& \text { and } \left.\left(\omega_{3}, \omega_{4}\right)_{i} \text { is a shock wave }\right\} .
\end{aligned}
$$

With this definition of $Q(\Delta)$ we have from Lemmas 3.1 and 3.2 and results on the interaction of elementary waves for conservation laws, $[3,8]$, that

$$
\alpha_{i}=\left(\omega_{1}, \omega_{2}\right)_{i}+\left(\omega_{3}, \omega_{4}\right)_{i}+O(1) Q(\Delta),
$$

where $\alpha_{i}$ is the strength of the $i$-wave leaving $\Delta$.

Suppose that (5.4) and (5.5) have been shown for $J=J_{1}$ and $J_{2}$ is an immediate successor of $J_{1}, \Delta$ the diamond between them. According to (5.6), waves may change their strengh only due to linear combining or cancelling, and nonlinear interaction as measured by $Q$. Since the only wave crossing 0 is the 1 -shock wave with strength $\alpha$ and issued from $\left(x_{*}, 0\right)$, it follows that the strength of this shock wave as it crosses $J_{1}$ is of the strength $\alpha_{0}+O(1) Q\left(\Lambda_{1}\right)$ and all other waves have a total strength $O(1) Q\left(\Lambda_{1}\right)$, where $\left(\Lambda_{1}\right)$ is the region below $J_{1}$. It follows from the induction hypothesis $(5.5)$ that

$$
Q\left(\Lambda_{1}\right) \leqq 2[Q(0)-Q(J)] \leqq 2 \alpha_{0} \int_{x_{*}}^{x_{1}}|c(x)| d x,
$$

where $x=x_{1}$ is the position of the relative shock wave as it crosses $J_{1}$. Thus, in particular, the relative strong shock wave has strength

$$
\alpha_{*}=\alpha_{0}\left[1+O(1) \int_{x_{*}}^{x_{1}}|c(x)| d x\right] \geqq \frac{\alpha_{0}}{2}
$$


as it crosses $J_{1}$. The speed of this shock wave, according to Lemma 3.2 and (5.7), is

$$
\begin{aligned}
\sigma\left(x_{1}\right) & \geqq \sigma\left(x_{*}\right)+1 / 2 d \int_{x_{*}}^{x_{1}}|c(y)| d y+O(1) Q\left(\Lambda_{1}\right) \\
& \geqq \sigma\left(x_{*}\right)+\frac{1}{3} d \int_{x_{*}}^{x_{1}}|c(y)| d y .
\end{aligned}
$$

Note that $\sigma\left(x_{*}\right)$ is assumed to be positive (though arbitrarily small) and a wave with positive speed may travel only to the right according to the scheme introduced in the previous section. Consequently, $x_{*} \geqq x_{1}$ and (5.9) implies by induction that the relative strong shock wave always has positive speed at least up to the point when it crosses $J_{1}$. We now establish (5.4) and (5.5) for $J=J_{2}$. Based on the above description of the relative strong 1-shock wave we know that 1-waves to the right (left) of this shock wave have negative (positive) speed. This, along with estimate (5.6), imply that

$$
\begin{gathered}
F\left(J_{2}\right)-F\left(J_{1}\right) \leqq-1 / 2 Q(\Delta) \leqq 0, \\
Q\left(J_{2}\right)-Q\left(J_{1}\right) \leqq-1 / 2 Q(\Delta),
\end{gathered}
$$

whence we have easily (5.4) and (5.5) for $J=J_{2}$. Actually, the functionals $F(J)$ and $Q(J)$ were defined so that these two crucial estimates hold (see also [8]). Details are left to the reader.

To finish the proof of the theorem, we show that the relatively strong shock wave leaves the region $x \in(0,1)$ in finite time so that the solution becomes a small perturbation of a stable wave pattern as studied in the previous theorem. We know from (5.9) that the speed of the shock wave is always positive. It remains to show that as a result of the equidistributedness of the sequence $\left\{a_{k}\right\}$ and (5.9), the time limit for the shock to leave $x \in(0,1)$ is independent of the mesh length $\Delta x=r$. Assume for the moment that the shock wave propagates along a continuous curve (which would be the case if the second scheme of the previous section were used). Let $x=x(t)$ be the position of the shock at time $t$; then $x^{\prime}(t)=\sigma(x)$ and we have from (5.9):

$$
x^{\prime \prime}(t) \geqq 1 / 3 d c(x(t)) x^{\prime}(t)
$$

For the approximate solution, the above estimate holds modulo an error which depends on how well equidistributed the sequence $\left\{a_{k}\right\}$ is and goes to zero as $\Delta x=r$ tends to zero, [7]. Thus for simplicity we may argue with the above inequality. Since $d$ is a positive constant, $c(x)$ is positive for $0<x<1, x(0) \in(0,1)$, $x^{\prime}(0)$ is positive (though it may be arbitrarily small), the inequality implies that

$$
x(t) \geqq x^{\prime}(0) \int_{0}^{t} \exp \left[\frac{d}{3} \int_{0}^{t} c(x(s)) d s\right] d z
$$

and so there exists a finite time $t_{*}$ independent of $\Delta x=r$ such that $x(t) \geqq 1$ for $t \geqq t_{*}$. In other words, the relative strong shock wave leaves the region $0<x<1$ before finite time $t_{*}$. This completes the proof of the theorem. Q.E.D. 


\section{Stable Flows}

We already know that a noninteracting wave pattern containing no standing shock wave is stable and a flow along a contracting duct which contains a standing shock wave is unstable. To complete the present study it remains to show that a flow with a standing shock wave along an expanding duct is stable. Since for an expanding duct, an asymptotic (i.e. noninteracting) wave pattern depends uniquely and smoothly on its end states at $x= \pm \infty,[10]$, it suffices to show that a small perturbation of a flow with a standing shock wave tends to an asymptotic wave pattern as time tends to infinity. Throughout this section we assume:

$$
\begin{array}{ll}
c(x) \equiv-\frac{a^{\prime}(x)}{a(x)}<0 & \text { for } 0<x<1, \\
c(x)=0 & \text { otherwise. }
\end{array}
$$

Theorem 6.1. Suppose that $\omega(x, 0)$ contains a 1-shock wave $\left(\omega_{-}, \omega_{+}\right)$at $x_{*} \in(0,1)$ with $\lambda_{1}\left(\omega_{-}\right)<0<\lambda_{1}\left(\omega_{+}\right)$, and both $|c(x)|$ and $\left|\sigma\left(\omega_{-}, \omega_{+}\right)\right|$are small compared to the strength $\alpha$ of the wave $\left(\omega_{-}, \omega_{+}\right)$. Suppose also that $\omega(x, 0)$ is a small perturbation in total variation of standing waves for $-\infty<x<x_{*}$ and for $x_{*}<x<\infty$. Then there exists a solution $\omega(x, t)$ of $(1.1)$ which tends to a noninteracting wave pattern as $t$ tends to infinity.

Proof. The difference between the situation here and that in Theorem 5.2 is that although there still exists a relatively strong shock wave for all time, this shock wave, unlike the corresponding one in Theorem 5.2, stays nearby the unperturbed standing shock wave. Consequently, we have to show that the shock wave decelerates in general and so would not cause too strong nonlinear interactions if it were to oscillate along the expansion portion of the duct. For this we define a different nonlinear functional $F(J)$ as follows: Suppose that the shock wave has speed $\sigma=\sigma(t)$, strength $\alpha$ and position $x=h_{0} r=h_{0}(t) r$ when it crosses $J$ (at time $t$ ). We set

$$
F(J) \equiv L(J)+K\left\{Q_{1}(J)+Q_{2}(J)+Q_{3}(J)+Q_{d}(J)\right\}
$$

where $L$ and $Q$ 's are the same as those in the previous section except that $Q_{1}^{c}(J)$ is set to be zero and $L(J)$ is redefined as

$$
\left.L(J) \equiv K|\sigma|+\sum|\alpha|: \alpha \text { the strength of any wave crossing } J\right\} .
$$

The proof of uniform boundedness of the total variation in $x$ of the approximate solution $\omega_{r}(x, t)$ is similar to the proof of Theorem 5.2. The notable difference here is that since we do not know a priori the sign of the speed of the relative strong shock wave, we cannot foresee the potential interaction of the shock with the geometry of the duct (which was taken care of by $Q_{1}^{c}$ in Theorem 5.2). Instead, we notice from Lemma 3.2 that when the shock moves between $x=0$ and $x=1$, where the duct is not uniform, it tends to decelerate and so $|\sigma|$ tends to decrease. This shows that the first part of $L(J)$ tends to decrease. This is sufficient to dominate the increase in the remaining parts of $F(J)$ as the result of the nonlinear interaction of the relatively strong shock wave with the duct. Therefore, in palce of (5.5), we have

$$
Q(\Lambda) \leqq O(1) \sigma(0)+2\{Q(0)-Q(J)\} .
$$


The above statements are shown using Lemmas 3.1, 3.2 and analogous arguments used in the proof of Theorem 5.2; details are omitted.

We now study the asymptotic behavior of the solution. Given any $\varepsilon>0$, it follows from (6.2) that there exists $T=T(\varepsilon)$ such that

$$
Q\{t \geqq T\} \leqq \varepsilon .
$$

By choosing $M=M(\varepsilon)$ sufficiently large we have

$$
\text { total variation }\{\{u(x, T):|x| \geqq M\} \leqq \varepsilon .
$$

Through $(M, T)$ and $(-M, T)$ we draw generalized $i$-characteristic curves $\chi_{i}^{+}$and $\chi_{i}^{-}, i=1,2,3$ which travel either with $i$-characteristic speed or $i$-discontinuity speed $[4,7]$. The region between $\chi_{i}^{+}$and $\chi_{i}^{-}$is denoted by $\Omega_{i}$. Due to the strict hyperbolicity of the system, $\chi_{i}^{ \pm}$do not intersect $\chi_{j}^{ \pm}, i \neq j$, after finite time $T_{0}>T$. After time $T_{0}$, the $i$-waves outside $\Omega_{i}$ are either produced by interaction or issued from $(x, T),|x| \geqq M$, and so (6.3) and (6.4) imply that the total amount of $i$-waves after time $T_{0}$ and outside $\Omega_{i}$ is $O(1) \varepsilon$. Thus in $\Omega_{i}$, $i$-waves cancel and combine and behave $(\bmod O(1) \varepsilon)$ like waves for scalar conservation law (Sect. 10, [8]). Consequently, modulo the error $O(1) \varepsilon 3$-waves tend either to a 3 -shock wave or a 3 -rarefaction wave, 2 -waves tend to a traveling wave and 1-waves tend to a shock wave due to the presence of the relative strong shock wave in the solution. Moreover, outside the 1 -shock wave and in the region $0<x<1$, the solution equals $(\bmod O(1) \varepsilon)$ standing waves. To show the closeness of such elementary waves to a noninteracting wave pattern it remains to show that when the 1-shock wave stays in the region $0<x<1$ its speed is close to zero. In fact we will show that in this case its speed is $O(1) \sqrt{\varepsilon}$ after time $T_{0}+\frac{2+\left|x_{0}\right|}{\varepsilon}$ where $x=x_{0}$ is the position of the 1 -shock wave at time $T_{0}$. For this we first note that the speed $\sigma(t)$ of the shock wave at time $t$ is a Lipschitz function of $t(\bmod O(1) \varepsilon)$ in the following sense: By Lemma 3.2 we have for $t_{2}>t_{1}>T_{0}$

$$
\begin{aligned}
\left|\sigma\left(t_{2}\right)\right| & \leqq\left|\sigma\left(t_{1}\right)\right|+O(1) Q\left\{t \in\left(t_{1}, t_{0}\right)\right\} \\
& =\left|\sigma\left(t_{1}\right)\right|+O(1) \varepsilon .
\end{aligned}
$$

Thus if $|\sigma(\bar{t})| \leqq \sqrt{\varepsilon}$ for some $\bar{t} \epsilon\left(T_{0}, T_{0}+\frac{2+\left|x_{0}\right|}{\varepsilon}\right)$ then $|\sigma(t)| \leqq \sqrt{\varepsilon}+O(1) \varepsilon \leqq 2 \sqrt{\varepsilon}$ for $t \geqq t_{0}$. It remains to show that if $|\sigma(t)| \geqq \sqrt{\varepsilon}$ for all $t \in\left(T_{0}, T_{0}+\frac{\left|x_{0}\right|+1}{\varepsilon}\right)$ then the 1 -shock wave does not stay in the region $0<x<1$ after time $T_{0}+\frac{\left|x_{0}\right|+2}{\varepsilon}$. In fact it is clear that when the speed of the one shock is larger than $\sqrt{\varepsilon}$ (or less than $-\sqrt{\varepsilon}$ ) for all $t \in\left(T_{0}, T_{0}+\frac{\left|x_{0}\right|+1}{\varepsilon}\right)$ then at time $T_{0}+\frac{\left|x_{0}\right|+1}{\varepsilon}$ the position of the shock wave is to the right of $x=1$ (or left of $x=0$ ) and by (6.4) its speed after that time is $\sqrt{\varepsilon}-O(1) \varepsilon \geqq 1 / 2 \sqrt{\varepsilon}[$ or $-\sqrt{\varepsilon}+O(1) \varepsilon \leqq-1 / 2 \sqrt{\varepsilon}]$ and always stays in the region $x \geqq 1$ (or $x<0$ ) for any larger time. This completes the arguments. 
When the perturbed data $\omega(x, 0)$ is equal to constant states both to the right and to the left of a finite interval, the convergence of the solution $\omega(x, t)$ to noninteracting wave patterns is of algebraic rates. For this more precise estimates are needed, we will not pursue that here (cf. $[6,8])$. This completes the proof of the theorem. Q.E.D.

\section{References}

1. Bethe, H.: Report on the theory of shock waves for an arbitrary equation of states, U.S. Dept. of Commerce Report No. PB-32189, Clearing house for Federal Scientific and Technical Information, 1942

2. Courant, R., Friedrichs, K.O.: Supersonic flow and shock waves. New York: Interscience 1948

3. Glimm, J.: Solutions in the large for nonlinear hyperbolic systems of equations. Commun. Pure Appl. Math. 18, 697-715 (1965)

4. Glimm, J., Lax, P.D.: Decay of solutions of systems of nonlinear hyperbolic conservation laws. Memoirs, Am. Math. Soc. 101 (1970)

5. Liu, T.-P.: Shock waves in the nonisentropic gas flow. J. Diff. Eq. 22, 442-452 (1976)

6. Liu, T.-P.: Linear and nonlinear large-time behavior of solutions of general systems of hyperbolic conservation laws. Commun. Pure Appl. Math. 30, 767-796 (1977)

7. Liu, T.-P.: Deterministic version of the Glimm scheme. Commun. Math. Phys. 57, 135-148 (1977)

8. Liu, T.-P.: Quasilinear hyperbolic systems. Commun. Math. Phys. 68, 141-172 (1979)

9. Liu, T.-P.: System of quasilinear hyperbolic partial differential equations. Proc. in Symposium on Trends in Appl. of Pure Math. to Mech., Knops (ed.). Putnam 1981

10. Liu, T.-P.: Transonic gas flows in a variable area duct. Arch. Rat. Mech. Anal. (to appear)

11. Weyl, H. : Shock waves in arbitrary fluids. Commun. Pure Appl. Math. 2, 103-122 (1949)

12. Whitham, B.: Linear and nonlinear waves. New York: John Wiley 1974

Communicated by J. Glimm

Received June 18, 1981 